\title{
Best possible sufficient conditions for the Fourier transform to satisfy the Lipschitz or Zygmund condition
}

by

\author{
FERENC MóRICZ (Szeged)
}

\begin{abstract}
We consider complex-valued functions $f \in L^{1}(\mathbb{R})$, and prove sufficient conditions in terms of $f$ to ensure that the Fourier transform $\hat{f}$ belongs to one of the Lipschitz classes $\operatorname{Lip}(\alpha)$ and $\operatorname{lip}(\alpha)$ for some $0<\alpha \leq 1$, or to one of the Zygmund classes $\operatorname{Zyg}(\alpha)$ and $\operatorname{zyg}(\alpha)$ for some $0<\alpha \leq 2$. These sufficient conditions are best possible in the sense that they are also necessary in the case of real-valued functions $f$ for which either $x f(x) \geq 0$ or $f(x) \geq 0$ almost everywhere.
\end{abstract}

1. Introduction. We consider complex-valued functions $f: \mathbb{R} \rightarrow \mathbb{C}$ which are integrable in Lebesgue's sense over $\mathbb{R}:=(-\infty, \infty)$, in symbols: $f \in L^{1}(\mathbb{R})$. As is well known, the Fourier transform of $f$ defined by

$$
\hat{f}(t):=\frac{1}{2 \pi} \int_{\mathbb{R}} f(x) e^{-i t x} d x, \quad t \in \mathbb{R},
$$

is a continuous function and $\hat{f}(t) \rightarrow 0$ as $|t| \rightarrow \infty$. For more information see, e.g., [2, Chapter I].

We recall that $\hat{f}$ is said to satisfy the Lipschitz condition of order $\alpha>0$, in symbols: $\hat{f} \in \operatorname{Lip}(\alpha)$, if

$$
|\hat{f}(t+h)-\hat{f}(t)| \leq C h^{\alpha} \quad \text { for all } t \in \mathbb{R} \text { and } h>0,
$$

where the constant $C$ does not depend on $t$ or $h$. Furthermore, $\hat{f}$ is said to belong to the little Lipschitz class $\operatorname{lip}(\alpha)$ for some $\alpha>0$ if

$$
\lim _{h \rightarrow 0} h^{-\alpha}[\hat{f}(t+h)-\hat{f}(t)]=0 \quad \text { uniformly in } t \in \mathbb{R} .
$$

Since $\hat{f}$ is bounded on $\mathbb{R}$ and vanishes at $\pm \infty$, it is enough to require the fulfillment of $(1.2)$ for $0<h \leq 1$.

2010 Mathematics Subject Classification: Primary 42A38; Secondary 26A16.

Key words and phrases: Fourier transform, best possible sufficient conditions, classical function classes $\operatorname{Lip}(\alpha), \operatorname{lip}(\alpha), \operatorname{Zyg}(\alpha)$ and $\operatorname{zyg}(\alpha)$. 
We recall that the Fourier transform $\hat{f}$ is said to satisfy the Zygmund condition of order $\alpha>0$, in symbols: $\hat{f} \in \operatorname{Zyg}(\alpha)$, if

$$
|\hat{f}(t+h)-2 \hat{f}(t)+\hat{f}(t-h)| \leq C h^{\alpha} \quad \text { for all } t \in \mathbb{R} \text { and } h>0,
$$

where the constant $C$ does not depend on $t$ or $h$. Furthermore, $f$ is said to belong to the little Zygmund class $\operatorname{zyg}(\alpha)$ for some $\alpha>0$ if

$$
\lim _{h \rightarrow 0} h^{-\alpha}[\hat{f}(t+h)-2 \hat{f}(t)+\hat{f}(t-h)]=0 \quad \text { uniformly in } t \in \mathbb{R} .
$$

Again, it is enough to require the fulfillment of (1.3) for $0<h \leq 1$.

It is well known (see, e.g., [1, Chapter 2] or [3, Chapter 2, §3]) that if $\hat{f} \in \operatorname{lip}(1)$, in particular if $\hat{f} \in \operatorname{Lip}(\alpha)$ for some $\alpha>1$, then $\hat{f} \equiv 0$. Furthermore, if $\hat{f} \in \operatorname{zyg}(2)$, in particular if $\hat{f} \in \operatorname{Zyg}(\alpha)$ for some $\alpha>2$, then $\hat{f} \equiv 0$.

2. Main results. Our main results are formulated in the following four theorems.

THEOREM 1.

(i) Suppose $f: \mathbb{R} \rightarrow \mathbb{C}$ is such that $f \in L_{\text {loc }}^{1}(\mathbb{R})$. If for some $0<\alpha \leq 1$,

$$
\int_{|x|<y}|x f(x)| d x=O\left(y^{1-\alpha}\right) \quad \text { for all } y>0,
$$

then $f \in L^{1}(\mathbb{R})$ and $\hat{f} \in \operatorname{Lip}(\alpha)$.

(ii) Conversely, suppose $f \in L^{1}(\mathbb{R})$ and $x f(x) \geq 0$ for almost every $x \in \mathbb{R}$. If $\hat{f} \in \operatorname{Lip}(\alpha)$ for some $0<\alpha \leq 1$, then condition (2.1) holds.

THEOREM 2.

(i) Suppose $f: \mathbb{R} \rightarrow \mathbb{C}$ is such that $f \in L_{\text {loc }}^{1}(\mathbb{R})$. If for some $0<\alpha \leq 2$,

$$
\int_{|x|<y} x^{2}|f(x)| d x=O\left(y^{2-\alpha}\right) \quad \text { for all } y>0,
$$

then $f \in L^{1}(\mathbb{R})$ and $\hat{f} \in \operatorname{Zyg}(\alpha)$.

(ii) Conversely, suppose $f \in L^{1}(\mathbb{R})$ and $f(x) \geq 0$ for almost every $x \in \mathbb{R}$. If $\hat{f} \in \operatorname{Zyg}(\alpha)$ for some $0<\alpha \leq 2$, then condition (2.2) holds.

Modifying the proofs of Theorems 1 and 2, in Section 4 we obtain the following two theorems.

TheORem 3. In case $0<\alpha<1$, both statements in Theorem 1 remain valid if the right-hand side in (2.1) is replaced by $o\left(y^{1-\alpha}\right)$ as $y \rightarrow \infty$, and $f \in \operatorname{Lip}(\alpha)$ is replaced by $f \in \operatorname{lip}(\alpha)$. 
THEOREM 4. In case $0<\alpha<2$, both statements in Theorem 2 remain valid if the right-hand side in $(2.2)$ is replaced by $o\left(y^{2-\alpha}\right)$ as $y \rightarrow \infty$, and $f \in \operatorname{Zyg}(\alpha)$ is replaced by $f \in \operatorname{zyg}(\alpha)$.

3. Auxiliary results. In this section, we consider nonnegative-valued, measurable functions $g$ defined on $\mathbb{R}_{+}:=[0, \infty)$. We will prove two lemmas, which are of interest in themselves.

LEMMA 1.

(i) If $\delta>\gamma \geq 0$ and

$$
\int_{0}^{y} u^{\delta} g(u) d u=O\left(y^{\gamma}\right) \quad \text { for all } y>0,
$$

then $g \in L^{1}(y, \infty)$ and

$$
\int_{y}^{\infty} g(u) d u=O\left(y^{\gamma-\delta}\right) \quad \text { for all } y>0 .
$$

(ii) Conversely, if $\delta \geq \gamma>0$ and condition (3.2) holds, then condition (3.1) also holds.

We note that Lemma 1 fails in the endpoint cases not included above. For example, if $\delta=\gamma>0$ in (i), then for $g(u):=u^{-1}$ condition (3.1) is satisfied, while (3.2) is not. If $\delta>\gamma=0$ in (ii), then for $g(u):=u^{-1-\delta}$ condition (3.2) is satisfied, while (3.1) is not.

Proof of Lemma 1. (i) By (3.1), there exists a constant $C=C(g)$ such that for all $y>0$,

$$
y^{\delta} \int_{y}^{2 y} g(u) d u \leq \int_{y}^{2 y} u^{\delta} g(u) d u \leq C(2 y)^{\gamma},
$$

whence it follows that

$$
\int_{y}^{2 y} g(u) d u \leq 2^{\gamma} C y^{\gamma-\delta},
$$

and since $\gamma<\delta$, we conclude that

$$
\begin{aligned}
\int_{y}^{\infty} g(u) d u & \leq 2^{\gamma} C y^{\gamma-\delta} \sum_{m=0}^{\infty} \int_{2^{m} y}^{2^{m+1} y} g(u) d u \\
& \leq 2^{\gamma} C y^{\gamma-\delta} \sum_{m=0}^{\infty} 2^{m(\gamma-\delta)}=O\left(y^{\gamma-\delta}\right), \quad y>0 .
\end{aligned}
$$

This proves (3.2). 
(ii) By (3.2), there exists another constant $C=C(g)$ such that for all $y>0$,

$$
\int_{y / 2}^{y} u^{\delta} g(u) d u \leq y^{\delta} \int_{y / 2}^{y} g(u) d u \leq 2^{\delta-\gamma} C y^{\gamma},
$$

and since $\gamma>0$, we conclude that

$$
\begin{aligned}
\int_{0}^{y} u^{\delta} g(u) d u & =\sum_{m=-\infty}^{0} \int_{2^{m-1} y}^{2^{m} y} u^{\delta} g(u) d u \\
& \leq 2^{\delta-\gamma} C y^{\gamma} \sum_{m=-\infty}^{0} 2^{m \gamma}=O\left(y^{\gamma}\right), \quad y>0 .
\end{aligned}
$$

This proves (3.1)

Modifying the proof of Lemma 1, we obtain

LEMMA 2.

(i) If $\delta>\gamma>0$ and

$$
\int_{0}^{y} u^{\delta} g(u) d u=o\left(y^{\gamma}\right) \quad \text { as } y \rightarrow \infty,
$$

then $g \in L^{1}(y, \infty)$ for large enough $y$ and

$$
\int_{y}^{\infty} g(u) d u=o\left(y^{\gamma-\delta}\right) \quad \text { as } y \rightarrow \infty .
$$

(ii) Conversely, if $\delta>\gamma>0, u^{\delta} g(u) \in L_{\text {loc }}^{1}\left(\mathbb{R}_{+}\right)$, and condition (3.8) holds, then condition also holds.

We note that the endpoint case $\delta>\gamma=0$ in (i) makes no sense, unless $g(u)=0$ almost everywhere, since the left-hand side in (3.7) is an increasing function of $y$. In the other endpoint case $\delta=\gamma \geq 0$, both (3.7) and (3.8) are trivially satisfied if $g \in L^{1}(\mathbb{R})$.

Proof of Lemma 2. (i) By (3.7), for every $\varepsilon>0$ there exists $y_{0}=y_{0}(\varepsilon)$ such that for all $y \geq y_{0},(3.3)$ is satisfied with $\varepsilon$ in place of $C$. Analogously to (3.4), it follows that

$$
\int_{y}^{\infty} g(u) d u \leq 2^{\gamma} \varepsilon y^{\gamma-\delta} \sum_{n=0}^{\infty} 2^{m(\gamma-\delta)}, \quad y \geq y_{0} .
$$

Since $\delta>\gamma$ and $\varepsilon>0$ is arbitrary, this proves (3.8). 
(ii) By (3.8), for every $\varepsilon>0$ there exists another $y_{0}=y_{0}(\varepsilon)$ such that for all $y \geq y_{0},(3.5)$ is satisfied with $\varepsilon$ in place of $C$, that is,

$$
\int_{y / 2}^{y} u^{\delta} g(u) d u \leq 2^{\delta-\gamma} \varepsilon y^{\gamma}, \quad y \geq y_{0} .
$$

Due to the assumption $u^{\delta} g(u) \in L_{\mathrm{loc}}^{1}\left(\mathbb{R}_{+}\right)$, there exists $y_{1}=y_{1}\left(\varepsilon, y_{0}\right)>$ $2 y_{0}$ such that

$$
\int_{0}^{y_{0}} u^{\delta} g(u) d u \leq \varepsilon y_{1}^{\gamma}
$$

Given any $y \geq y_{1}$, there exists an integer $m_{0}=m_{0}\left(y_{1}\right) \leq-1$ for which

$$
2^{-m_{0}-1} y<y_{0} \leq 2^{m_{0}} y
$$

Now, by (3.9) and (3.10), we conclude (cf. (3.6)) that for all $y \geq y_{1}$ we have

$$
\begin{aligned}
\int_{0}^{y} u^{\delta} g(u) d u & \leq\left\{\int_{0}^{y_{0}}+\sum_{m=-m_{0}}^{0} \int_{2^{m-1} y}^{2^{m} y}\right\} u^{\delta} g(u) d u \\
& \leq \varepsilon y_{1}^{\gamma}+\sum_{m=-m_{0}}^{0} 2^{\delta-\gamma} \varepsilon\left(2^{m} y\right)^{\gamma} \leq \varepsilon y^{\gamma}\left(1+2^{\delta-\gamma} \sum_{m=-m_{0}}^{0} 2^{m \gamma}\right) .
\end{aligned}
$$

Since $\gamma>0$ and $\varepsilon>0$ is arbitrary, this proves (3.7).

\section{Proofs of theorems}

Proof of Theorem 1. (i) For any $t \in \mathbb{R}$ and $h>0$, by (1.1) we have

$$
\begin{aligned}
2 \pi|\hat{f}(t+h)-\hat{f}(t)| & =\left|\int_{\mathbb{R}} f(x) e^{-i t x}\left(e^{-i h x}-1\right) d x\right| \\
& \leq\left\{\int_{|x|<1 / h}+\int_{|x|>1 / h}\right\}|f(x)|\left|e^{-i h x}-1\right|=: I_{h}+J_{h},
\end{aligned}
$$

say. Since

$$
\left|e^{-i h x}-1\right|=\left|2 \sin \frac{h x}{2}\right| \leq \min \{2, h|x|\},
$$

by (2.1) we estimate as follows:

$$
\left|I_{h}\right| \leq h \int_{|x|<1 / h}|x f(x)| d x=h O\left(\left(\frac{1}{h}\right)^{1-\alpha}\right)=O\left(h^{\alpha}\right) .
$$

Applying Lemma 1(i) in the case of (2.1), we find that

$$
\left|J_{h}\right| \leq 2 \int_{|x|>1 / h}|f(x)| d x=O\left(\left(\frac{1}{h}\right)^{-\alpha}\right)=O\left(h^{\alpha}\right) .
$$

Combining (4.1)-(4.3) gives $\hat{f} \in \operatorname{Lip}(\alpha)$. 
(ii) Assume $\hat{f} \in \operatorname{Lip}(\alpha)$ for some $0<\alpha \leq 1$. By (1.1), we have

$$
2 \pi|\hat{f}(t)-\hat{f}(0)|=\left|\int_{\mathbb{R}} f(x)\left(e^{-i t x}-1\right) d x\right| \leq C t^{\alpha}, \quad t>0,
$$

where the constant $C$ does not depend on $t$. Taking only the imaginary part of the integral between the absolute value bars, we even have

$$
\left|\int_{\mathbb{R}} f(x) \sin t x d x\right| \leq C t^{\alpha}, \quad t>0 .
$$

We may integrate the integral in (4.4) with respect to $t$ over the interval $(0, h)$, where $h>0$. By Fubini's theorem, we obtain

$$
\left|\int_{\mathbb{R}} f(x) \frac{1-\cos h x}{x} d x\right|=\int_{\mathbb{R}} \frac{f(x)}{x} 2 \sin ^{2} \frac{h x}{2} d x \leq C \frac{h^{\alpha+1}}{\alpha+1},
$$

where the constant $C$ does not depend on $h$, and we took into account that $x f(x) \geq 0$. Using the well-known inequality

$$
\sin u \geq \frac{2}{\pi} u \quad \text { for } 0 \leq u \leq \pi / 2,
$$

it follows from (4.5) that

that is,

$$
\frac{2 h^{2}}{\pi^{2}} \int_{|x|<1 / h} x f(x) d x \leq C \frac{h^{\alpha+1}}{\alpha+1}
$$

$$
\int_{|x|<1 / h} x f(x) d x \leq \frac{C \pi^{2}}{2(\alpha+1)} h^{\alpha-1}=O\left(\left(\frac{1}{h}\right)^{1-\alpha}\right), \quad h>0 .
$$

This proves (2.1) with $y:=1 / h, h>0$.

Proof of Theorem 3. It runs along the same lines as the proof of Theorem 1, using Lemma 2 instead of Lemma 1. The details are left to the reader.

Proof of Theorem 2. (i) For any $t \in \mathbb{R}$ and $h>0$, by (1.1) we have

$$
\begin{aligned}
& 2 \pi|\hat{f}(t+h)-2 \hat{f}(t)+\hat{f}(t-h)| \\
& \quad=\left|\int_{\mathbb{R}} f(x) e^{-i t x}\left(e^{-i h x}-2+e^{i h x}\right) d x\right| \\
& \quad \leq\left\{\int_{|x|<1 / h}+\int_{|x|>1 / h}\right\}|f(x)|\left|e^{-i h x}-2+e^{i h x}\right| d x=: I_{h}+J_{h},
\end{aligned}
$$

say. Since

$$
\left|e^{-i h x}-2+e^{i h x}\right|=|2(\cos h x-1)|=4 \sin ^{2} \frac{h x}{2} \leq \min \left\{4, h^{2} x^{2}\right\},
$$

by (2.2) we estimate as follows: 


$$
\left|I_{h}\right| \leq h^{2} \int_{|x|<1 / h} x^{2}|f(x)| d x=h^{2} O\left(\left(\frac{1}{h}\right)^{2-\alpha}\right)=O\left(h^{\alpha}\right) .
$$

Applying Lemma 1(i) in the case of (2.2), we find that

$$
\left|J_{h}\right| \leq 4 \int_{|x|>1 / h}|f(x)| d x=O\left(\left(\frac{1}{h}\right)^{-\alpha}\right)=O\left(h^{\alpha}\right) .
$$

Combining (4.7)-(4.9) gives $\hat{f} \in \operatorname{Zyg}(\alpha)$.

(ii) Assume $\hat{f} \in \operatorname{Zyg}(\alpha)$ for some $0<\alpha \leq 2$. By (1.1), we have

$$
\begin{aligned}
2 \pi|\hat{f}(h)-2 \hat{f}(0)+\hat{f}(-h)| & =\left|\int_{\mathbb{R}} f(x)(2 \cos h x-2) d x\right| \\
& =4 \int_{\mathbb{R}} f(x) \sin ^{2} \frac{h x}{2} d x \leq C h^{\alpha}, \quad h>0,
\end{aligned}
$$

where the constant $C$ does not depend on $h$, and we took into account that $f(x) \geq 0$. Making use of inequality (4.6), it follows from (4.10) that

$$
\frac{4 h^{2}}{\pi^{2}} \int_{|x|<1 / h} x^{2} f(x) d x \leq C h^{\alpha}
$$

that is,

$$
\int_{|x|<1 / h} x^{2} f(x) d x \leq \frac{C \pi^{2}}{4} h^{\alpha-2}=O\left(\left(\frac{1}{h}\right)^{2-\alpha}\right), \quad h>0 .
$$

This proves (2.2) with $y:=1 / h, h>0$.

Proof of Theorem 4. It is a repetition of the proof of Theorem 2 with appropriate modifications, using Lemma 2 instead of Lemma 1. The details are left to the reader.

\section{References}

[1] R. de Vore and G. G. Lorentz, Constructive Approximation, Springer, Berlin, 1993.

[2] E. M. Stein and G. Weiss, Introduction to Fourier Analysis on Euclidean Spaces, Princeton Univ. Press, 1971.

[3] A. Zygmund, Trigonometric Series, Vol. I, Cambridge Univ. Press, 1959.

Ferenc Móricz

Bolyai Institute

University of Szeged

Aradi vértanúk tere 1

H-6720 Szeged, Hungary

E-mail: moricz@math.u-szeged.hu 